\title{
Quality or quantity: is nutrient transfer driven more by symbiont identity and productivity than by symbiont abundance?
}

\author{
Christopher J Freeman ${ }^{1}$, Robert W Thacker ${ }^{1}$, David M Baker ${ }^{2,3}$ and Marilyn L Fogel ${ }^{2}$ \\ ${ }^{1}$ Department of Biology, University of Alabama at Birmingham, Birmingham AL, USA; ${ }^{2}$ Geophysical \\ Laboratory, Carnegie Institution of Washington, Washington, DC, USA and ${ }^{3}$ Smithsonian Institution, \\ National Museum of Natural History, Washington, DC, USA
}

By forming symbiotic interactions with microbes, many animals and plants gain access to the products of novel metabolic pathways. We investigated the transfer of symbiont-derived carbon and nitrogen to the sponges Aplysina cauliformis, Aplysina fulva, Chondrilla caribensis, Neopetrosia subtriangularis and Xestospongia bocatorensis, all of which host abundant microbial populations, and Niphates erecta, which hosts a sparse symbiont community. We incubated sponges in light and dark bottles containing seawater spiked with ${ }^{13} \mathrm{C}$ - and ${ }^{15} \mathrm{~N}$-enriched inorganic compounds and then measured ${ }^{13} \mathrm{C}$ and ${ }^{15} \mathrm{~N}$ enrichment in the microbial (nutrient assimilation) and sponge (nutrient transfer) fractions. Surprisingly, although most sponges hosting abundant microbial communities were more enriched in ${ }^{13} \mathrm{C}$ than $N$. erecta, only $N$. subtriangularis was more enriched in ${ }^{15} \mathrm{~N}$ than $N$. erecta. Although photosymbiont abundance varied substantially across species, ${ }^{13} \mathrm{C}$ and ${ }^{15} \mathrm{~N}$ enrichment was not significantly correlated with photosymbiont abundance. Enrichment was significantly correlated with the ratio of gross productivity to respiration (P:R), which varied across host species and symbiont phylotype. Because irradiance impacts $P: R$ ratios, we also incubated $A$. cauliformis in ${ }^{13} \mathrm{C}$-enriched seawater under different irradiances to determine whether symbiont carbon fixation and transfer are dependent on irradiance. Carbon fixation and transfer to the sponge host occurred in all treatments, but was greatest at higher irradiances and was significantly correlated with P:R ratios. Taken together, these results demonstrate that nutrient transfer from microbial symbionts to host sponges is influenced more by host-symbiont identities and P:R ratios than by symbiont abundance.

The ISME Journal (2013) 7, 1116-1125; doi:10.1038/ismej.2013.7; published online 14 February 2013 Subject Category: microbe-microbe and microbe-host interactions

Keywords: cyanobacteria; microbial symbionts; nutrient exchange; porifera; stable isotopes; symbiosis

\section{Introduction}

Symbioses between prokaryotes and their eukaryotic hosts are found across disparate taxa and within diverse environments ranging from deep-sea vents, coral reefs, terrestrial soils, marine and terrestrial invertebrates, and the mammalian gut (Boucher et al., 1982; Zilber-Rosenberg and Rosenberg, 2008; Rosenberg et al., 2010). In many cases, interactions between distantly related organisms are mutually beneficial, whereby the symbiont is provided a protected, nutrient-rich habitat in exchange for products of its metabolism (Venn et al., 2008; Vrijenhoek, 2010). These interactions can be highly

Correspondence: C Freeman, Smithsonian Marine Station at Fort Pierce, 701 Seaway Drive, Fort Pierce, FL 34949, USA.

E-mail: cjfre_freeman@yahoo.com

Received 4 May 2012; revised 20 December 2012; accepted 3 January 2013; published online 14 February 2013 coevolved, leading to adaptations such as vertical transmission of symbionts and reduced symbiont genomes (Nyholm and McFall-Ngai, 2004; Moya et al., 2008; Zilber-Rosenberg and Rosenberg, 2008; Thacker and Freeman, 2012).

In the marine environment, nutritional symbioses are exemplified by mutualisms between reef-building corals and dinoflagellates. Indeed, reef-building corals would be unlikely to grow and compete for substrates in oligotrophic waters without supplemental nutrition provided by their symbionts (Muscatine and Cernichiari, 1969; Muscatine et al., 1989). Other diverse groups of marine organisms also host microbial symbionts, but these interactions are less well understood (Taylor et al., 2007a, b; Venn et al., 2008; Webster and Blackall, 2009; Webster and Taylor, 2012; Thacker and Freeman, 2012). For instance, marine sponges can dominate benthic communities throughout the world (Becerro, 2008) and often host abundant and diverse 
microbial communities that are capable of complex metabolic pathways, including carbon fixation and nitrogen transformations (Taylor et al., 2007a, b; Webster et al., 2010; Siegl et al., 2011).

Two recent studies demonstrated that stable isotopes of carbon $\left({ }^{13} \mathrm{C}\right)$ and nitrogen $\left({ }^{15} \mathrm{~N}\right)$ could be used to investigate the transfer of symbiont carbon and nitrogen to the host sponge (Weisz et al., 2007; Freeman and Thacker, 2011). Isotope values of isolated sponge cell and microbial symbiont cell fractions revealed that the transfer of symbiont carbon and nitrogen differs across host species (Freeman and Thacker, 2011). Thus, while some sponge-microbe symbioses appear to be facultative mutualisms, other sponge hosts do not acquire the products of symbiont metabolism and form commensal interactions (Freeman and Thacker, 2011).

Inorganic compounds enriched in ${ }^{13} \mathrm{C}$ (for example, $\mathrm{NaH}^{13} \mathrm{CO}_{3}$ ) and ${ }^{15} \mathrm{~N}$ (for example, $\mathrm{Na}^{15} \mathrm{NO}_{3}$ ) can serve as metabolic 'tracers' to follow the transfer of autotrophic symbiont nutrition to a host (Tanaka et al., 2006). Because these compounds are assimilated via photosynthesis, host sponge cells become isotopically enriched through the transfer of enriched organic biomolecules from photosynthetic symbionts. To date, studies using this approach have conducted 'pulse-chase' experiments, whereby organisms are 'pulsed' in water containing enriched tracer for a short period of time and subsequently held in natural seawater for a 'chase' period, during which the transfer of enriched compounds is followed (Tanaka et al., 2006, Weisz et al., 2010). While informative, such experiments are most often conducted with a single species and include relatively lengthy chase periods (1-2 weeks), which limit the contemporaneous determination of differences among closely related species, and thus significant functional variation within taxonomic groups.

Although the transfer of symbiont-derived carbon and nitrogen varies across sponges that host dense microbial communities, and appears to be negligible in species hosting sparse microbial communities (Weisz et al., 2007; Freeman and Thacker, 2011), the factors shaping such variation remain unknown. For example, photosymbiont abundance, the gross productivity to respiration $(\mathrm{P}: \mathrm{R})$ ratio of the symbiosis (that is, whether photosymbionts can compensate for holobiont respiration) and symbiont phylotype also vary across host species (Wilkinson, 1983; Erwin and Thacker, 2007; 2008a; Freeman and Thacker, 2011). To determine whether these factors impact nutrient transfer, we coupled short-term light-dark bottle incubations with two tracer compounds $\left(\mathrm{NaH}^{13} \mathrm{CO}_{3}\right.$ and $\left.\mathrm{Na}^{15} \mathrm{NO}_{3}\right)$, followed by the separation of symbiont and host cell fractions (Freeman and Thacker, 2011) before stable isotope analysis. We compared carbon and nitrogen assimilation by microbial symbionts and nutrient transfer to the host to photosymbiont abundance, $\mathrm{P}: \mathrm{R}$ ratios (Wilkinson, 1983; Erwin and Thacker, 2008a) and photosymbiont identity (Erwin and Thacker, 2007, 2008b). Because $P: R$ is strongly influenced by irradiance within single species (Erwin and Thacker, 2008a), we also incubated Aplysina cauliformis in seawater spiked with $\mathrm{NaH}^{13} \mathrm{CO}_{3}$ under varying irradiances. This experimental design allowed us to determine whether symbiont carbon fixation and nutrient transfer increase with increasing P:R. We tested three hypotheses: $(1){ }^{13} \mathrm{C}$ and ${ }^{15} \mathrm{~N}$ enrichment of the microbial cell fraction (assimilation of inorganic compounds) and the sponge cell fraction (transfer of symbiont nutrition to the host) will be highest in sponges hosting dense symbiont communities, but will be negligible in Niphates erecta, which hosts only a sparse symbiont community; (2) variability in ${ }^{13} \mathrm{C}$ and ${ }^{15} \mathrm{~N}$ enrichment across species will be driven more by photosymbiont abundance than by the P:R ratio of the symbiosis; and (3) carbon assimilation and transfer in A. cauliformis will be highest at maximum irradiance and will be directly correlated to P:R ratios.

\section{Materials and methods}

Study organisms

Sponges were collected from shallow (2-4 m) reefs adjacent to the Smithsonian Tropical Research Institute in Bocas del Toro, Panama. Six focal species differed in their growth forms, symbiont phylotypes and reliance on symbiont nutrition. A. cauliformis, Aplysina fulva and Neopetrosia subtriangularis all host abundant microbial communities that are dominated by unicellular cyanobacteria (Erwin and Thacker, 2007); however, each of these hosts forms a unique relationship with its symbionts (Freeman and Thacker, 2011). The sponge $N$. erecta hosts a sparse community of nonphotosynthetic microbes (Erwin and Thacker, 2007; Weisz et al., 2007), and gains nutrients primarily by filter feeding (Freeman and Thacker, 2011); taken together, these characteristics indicate that this species is a suitable negative control in our experiment. While these four sponges have rope-like growth forms, the sponges Xestospongia bocatorensis and Chondrilla caribensis grow as encrusting cushions and host abundant filamentous and unicellular cyanobacterial symbionts, respectively (Erwin and Thacker, 2007; Thacker et al., 2007).

Five large individuals of each of these species were transported to aquaria, where each individual was divided into three replicate pieces. An initial piece was wrapped in aluminum foil and immediately frozen for initial measurements of natural abundance stable isotope ratios $\left(\delta^{13} \mathrm{C}\right.$ and $\left.\delta^{15} \mathrm{~N}\right)$ as well as chlorophyll $a$ (chl $a$ ) concentrations, while two pieces were used in the experimental incubations. Experimental pieces were incubated in flowthrough seawater tanks for at least $12 \mathrm{~h}$ overnight to allow sponges to acclimate and to ensure that only healthy individuals were used in the experiment. 
$\delta^{13} C$ and $\delta^{15} N$ tracer experiment

Before the experiment, $2.0 \mathrm{~g}$ of $98 \mathrm{at} \%{ }^{13} \mathrm{C} \mathrm{NaH}^{13} \mathrm{CO}_{3}$ $(1.18 \mathrm{~mm})$ and $200 \mathrm{mg}$ of 98 at $\%{ }^{15} \mathrm{~N} \quad \mathrm{Na}^{15} \mathrm{NO}_{3}$ $(0.117 \mathrm{~mm})$ were added to $20 \mathrm{l}$ of filtered $(0.70 \mu \mathrm{m}$ GF/F Whatman, GE Healthcare Life Sciences, Piscataway, NJ, USA) seawater collected from offshore. These compounds were used to trace autotrophic pathways from microbial symbionts to host sponges. Concentrations were selected based on preliminary data suggesting that these were the lowest concentrations of tracer that provided adequate signal during short-term incubations. This 'enriched seawater' was partitioned equally among 40, $500 \mathrm{ml}$ Nalgene HDPE bottles (20 light (translucent, with $\sim 50 \%$ of ambient light-transmitted) bottles and 20 dark (amber, with $0 \%$ of ambient light-transmitted) bottles). Initial dissolved oxygen (DO, $\mathrm{mg} \mathrm{l}^{-1}$ ) concentrations were measured using a NexSens (NexSens Technology, Inc., Alpha, $\mathrm{OH}$, USA) WQ-DO dissolved oxygen sensor. This experimental design separated replicates and species, but meant that incubations could be conducted with only four species at a time (five individuals of four species split among light and dark bottle treatments). For this reason, two sets of sponges were incubated on consecutive days (four species the first day and two the following day). Light and dark bottles were incubated under natural sunlight in a flow-through seawater tank (functioning as a constant temperature bath) for 6.4 and $5.9 \mathrm{~h}$ on the first and second days, respectively. Ambient irradiance and temperature was recorded every 2 min during these incubations using $\mathrm{HOBO}$ data loggers attached to the incubation tank and within light and dark bottles. At the end of the incubation, all bottles were removed from the tank and final DO concentrations were measured. Sponges were removed, blotted dry, weighed, wrapped in aluminum foil and frozen for future analyses. To ensure that only metabolically active tissue (organic matter) was included in these measurements, additional specimens of each species were collected for construction of standard regressions between blotted wet weights, dry weights and ash-free dry weights. Blotted wet weights and dry weights were normalized to ashfree dry weights using the species-specific standard curves.

\section{$\delta^{13} \mathrm{C}$ assimilation and transfer under varying irradiances}

Eight large $(\sim 50 \mathrm{~cm})$ individuals of $A$. cauliformis were collected at a $4 \mathrm{~m}$ deep reef near Smithsonian Tropical Research Institute Point, and returned to the laboratory. Each of these replicates was partitioned into five pieces (one for initial measurements and four experimental pieces); the experimental pieces were allowed to acclimate overnight in flow-through seawater aquaria as above. Translucent and dark bottles were filled with filtered $(0.70 \mu \mathrm{m} \mathrm{GF} / \mathrm{F}$ Whatman) seawater that was enriched with $\mathrm{NaH}^{13} \mathrm{CO}_{3}$, as above.
Experimental pieces were allocated to each of four treatments (dark (amber), light (translucent), lightly shaded (light bottle wrapped with two layers of shade cloth, approximately $50 \%$ of light bottle irradiance) and heavily shaded (light bottle wrapped with five layers of shade cloth, approximately $10 \%$ of light bottle irradiance)). Ambient and shaded irradiances were measured using $\mathrm{HOBO}$ data loggers (Onset) in lux and converted to $\mu \mathrm{mol}$ photons $\mathrm{m}^{-2} \mathrm{~s}^{-1} \mathrm{using}$ published conversion factors (http: //www.licor.com/) that were validated by simultaneous measurements from a HOBO data logger and a Licor light meter. Experimental irradiances were chosen because prior research demonstrated that sponges under the lightly shaded treatment were near the compensation point of photosynthesis, while sponges under the highly shaded treatment were below this compensation point (Erwin and Thacker, 2008a).

\section{$P: R$ ratios and chl $a$}

Net primary productivity was calculated for each light incubation bottle by subtracting the initial DO concentration from the final DO concentration; the same calculation was applied to dark bottles to yield respiration (R). Values of net primary productivity and $\mathrm{R}$ were corrected for ash-free dry weights and incubation time so that final values were expressed as $\mathrm{mg} \mathrm{O}_{2} \mathrm{l}^{-1} \mathrm{~g}^{-1} \mathrm{~h}^{-1}$. Gross primary productivity was calculated by subtracting $\mathrm{R}$ from net primary productivity for each individual. Net primary productivity, $\mathrm{R}$ and gross primary productivity values for each species are provided as Supplementary Information S1. Gross photosynthesis:respiration ratios (P:R) were calculated by dividing gross primary productivity by respiration. Because the experiments were conducted over the course of 2 days, $P: R$ values were corrected to account for differences in irradiance. Irradiance was measured every 2 min during the incubation period, and then averaged for each day's incubation period. $P: R$ values were analyzed using an analysis of covariance, with mean light intensity as the covariate, to obtain an adjusted least-squares mean and standard error for the P:R ratios of each species.

To estimate photosymbiont abundance within these sponges, we measured chl a concentrations using lyophilized samples of each piece of sponge, following methods described by Freeman and Thacker (2011). Although chl $a$ values can vary owing to the physiological condition of some symbionts, this metric is widely cited as the optimal method for quantifying photosymbiont abundance (Becerro and Paul, 2004; Sacristan-Soriano et al., 2011; Hill and Scott, 2012). Values were corrected for ash-free dry weights. Because mean chl $a$ values did not differ across treatment for each species (that is, values from initial samples and both treatments were not significantly different, as determined by a general linear model (GLM; Systat, version 11; Systat Software, Inc., Chicago, IL, USA) testing 
treatment nested within species $(P=0.84, \mathrm{~F}=0.58)$ and species $(P<0.001, \mathrm{~F}=237)$, the chl $a$ value for each species represents the combined mean of all subsamples from each species.

\section{Stable isotope analyses}

Symbiont and host cell fractions were separated from bulk sponge tissue in A. cauliformis, A. fulva, C. caribensis, N. erecta and N. subtriangularis using methods adapted from Freeman and Thacker (2011), provided as Supplementary Information S2. New methods were developed for $X$. bocatorensis, which hosts filamentous cyanobacterial symbionts; these new methods are also provided in Supplementary Information S2. Lyophilized microbial and sponge cell pellets were prepared for isotope analyses as described in Freeman and Thacker (2011). Stable isotope compositions were measured at the Geophysical Laboratory using a Thermo Delta V isotope ratio mass spectrometer (Thermo Scientific, West Palm Beach, FL, USA) coupled to a Carlo-Erba NC2500 elemental analyzer via a Conflo III opensplit interface. Mean precision of $\delta^{13} \mathrm{C}$ and $\delta^{15} \mathrm{~N}$ from seven or eight sets of duplicate samples from each treatment was $\pm 0.2 \%$ and $\pm 0.1 \%$ in initials, $\pm 0.23 \%$ and $\pm 2.25 \%$ in samples from the dark treatment. In the light treatment, samples from species other than $N$. subtriangularis had mean $\delta^{13} \mathrm{C}$ and $\delta^{15} \mathrm{~N}$ precision values of $\pm 15.8 \%$ and $\pm 3.8 \%$. Given that the observed magnitude of enrichment for $N$. subtriangularis was much higher than the other samples, its precision was calculated separately $\left(197 \%\right.$ and $209 \%$ for $\delta^{13} \mathrm{C}$ and $\delta^{15} \mathrm{~N}$, respectively). Isotope enrichment (expressed as APE (Atom Percent Excess)) was calculated using formulas adapted from Fry (2006), Tanaka et al. (2006) and Weisz et al. (2010):

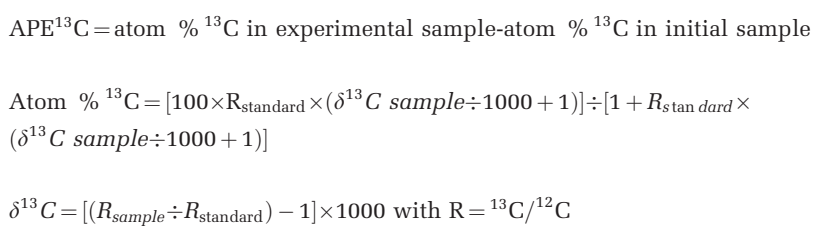

In experiments using tracer compounds, enrichment measured as APE assesses the increase in the atom percent of ${ }^{13} \mathrm{C}$ in experimental samples compared with the initial (or natural abundance) samples; thus, 'sample' refers to either the microbial or sponge cell fraction from an individual and 'standard' refers to values of international standards of Vienna Pee Dee Belemnite and atmospheric $\mathrm{N}_{2}$ for $\delta^{13} \mathrm{C}$ and $\delta^{15} \mathrm{~N}$, respectively (Fry, 2006). The formulas above are adapted for nitrogen by replacing ${ }^{15} \mathrm{~N}$ and ${ }^{14} \mathrm{~N}$ for ${ }^{13} \mathrm{C}$ and ${ }^{12} \mathrm{C}$.

\section{Data analysis}

All raw data for this manuscript have been deposited at Dryad. Mean $\mathrm{APE}^{13} \mathrm{C}$ and $\mathrm{APE}^{15} \mathrm{~N}$ values across treatments (light and dark bottles) were compared using GLMs, with treatment as the main factor and source individual as a blocking variable, for each fraction of each species. For these and all subsequent analyses, residuals were tested for normality and homogeneity of variances among groups. To determine if the microbial and host cell fractions of each species differed in $\mathrm{APE}^{13} \mathrm{C}$ or $\mathrm{APE}^{15} \mathrm{~N}$ values, GLMs, with fraction as the main factor and individual as a blocking variable, were analyzed for each species. Mean APE values of sponge and microbial fractions across the six species were compared using four separate analyses of variance (ANOVA), followed by pairwise comparisons across species using the Fisher's least-significant difference (LSD) test.

For P:R measurements, the adjusted least-squares means for each species from the analysis of covariance were compared using post hoc pairwise comparisons. Chl a concentrations across species were compared using a GLM testing the effects of species and treatment nested within species, followed by post hoc pairwise comparisons across species. Linear regressions determined whether trends in mean $\mathrm{APE}^{13} \mathrm{C}$ and $\mathrm{APE}^{15} \mathrm{~N}$ across species were related to photosymbiont abundance (measured as chl a concentrations) or productivity (measured as P:R ratios). All pairwise comparisons were conducted using the LSD test.

In the irradiance experiment with ${ }^{13} \mathrm{C}$ tracer, $\mathrm{P}: \mathrm{R}$ ratios across treatments were compared using a GLM testing the effect of treatment blocked by individual. Mean $\mathrm{APE}^{13} \mathrm{C}$ values of the two fractions within each treatment were compared with a GLM testing the effect of fraction blocked by individual. The mean $\mathrm{APE}^{13} \mathrm{C}$ values of each fraction across treatments were compared using a GLM testing the effect of treatment blocked by individual. The relationship between $\mathrm{APE}^{13} \mathrm{C}$ values and productivity was determined using a GLM testing the effects of individual and $P: R$ ratio on $A P E^{13} \mathrm{C}$. Multiple pairwise comparisons were conducted using an LSD test.

\section{Results and discussion}

${ }^{13} \mathrm{C}$ and ${ }^{15} \mathrm{~N}$ tracer experiment

Six species of marine sponges spanning diverse symbiotic interactions were incubated in $\mathrm{NaH}^{13} \mathrm{CO}_{3}$ and $\mathrm{Na}^{15} \mathrm{NO}_{3}$ to trace the transfer of symbiontderived carbon and nitrogen to the host. Our observed enrichment of ${ }^{15} \mathrm{~N}$ or ${ }^{13} \mathrm{C}$ in sponge cell fractions is direct evidence for the translocation of enriched biomolecules from the symbiont. Variations in the extent of uptake of ${ }^{15} \mathrm{~N}$ or ${ }^{13} \mathrm{C}$ could be due to the fact that sponges hosting abundant microbial communities (termed high microbial abundance (HMA) sponges) are generally thought to obtain large amounts of symbiont-derived nutrients compared with sponges hosting sparse symbiont communities (termed low microbial 
abundance sponges) (Hentschel et al., 2006; Taylor et al., 2007a). Minimal experimental evidence, however, supports this hypothesis (Thacker and Freeman, 2012).

Therefore, we originally hypothesized that HMA sponges would have higher ${ }^{13} \mathrm{C}$ and ${ }^{15} \mathrm{~N}$ enrichment than $N$. erecta, which lacks photosymbionts, contains bacteria that are most closely related to those found in the water column (Weisz et al., 2007), grows faster in the shade, and has natural abundance isotope values suggestive of a heavy reliance on filter feeding (Freeman and Thacker, 2011). Indeed, $\mathrm{APE}^{13} \mathrm{C}$ and $\mathrm{APE}^{15} \mathrm{~N}$ values in $N$. erecta were low regardless of whether they were incubated in a light or dark bottle (GLM: $P=0.064, \mathrm{~F}=0.26$ and $P=0.074, \mathrm{~F}=11.99$ for $\mathrm{APE}^{13} \mathrm{C}$ of microbial and sponge fractions, respectively, and $P=0.706, \mathrm{~F}=0.16$ and $P=0.26, \mathrm{~F}=2.38$ for $\mathrm{APE}^{15} \mathrm{~N}$ of microbial and sponge fractions, respectively; Figures 1 and 2), suggesting that inorganic $\mathrm{C}$ and $\mathrm{N}$ were largely inaccessible to this low microbial abundance sponge.

As expected, microbial and sponge cell fractions from almost all (except the sponge fraction of C. caribensis) of the HMA species were significantly more enriched in ${ }^{13} \mathrm{C}$ than the same fractions in $N$. erecta (Figure 1). In addition, there was significant variation in $\mathrm{APE}^{13} \mathrm{C}$ values of the microbial and sponge cell fractions across the HMA species held in light bottles (ANOVA: $P<0.001, \quad \mathrm{~F}=45.6$ and $P<0.001, \mathrm{~F}=15.43$ for microbial and sponge cell fractions, respectively), as A. cauliformis, A. fulva, $C$. caribensis and $X$. bocatorensis had similar values,

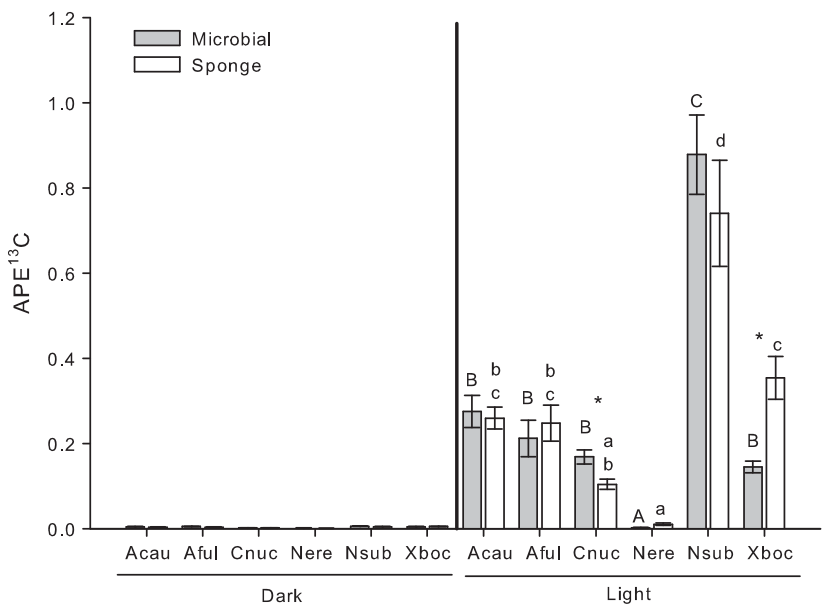

Figure 1 Mean ( \pm s.e.) $\mathrm{APE}^{13} \mathrm{C}$ values for microbial and sponge cell fractions from the sponges $A$. cauliformis (Acau), A. fulva (Aful), C. caribensis (Cnuc), N. erecta (Nere), N. subtriangularis (Nsub) and $X$. bocatorensis (Xboc) incubated in light (clear) and dark (amber) bottles. Values from the dark treatment are included for reference. Different letters within columns indicate statistically significant differences in the $\mathrm{APE}^{13} \mathrm{C}$ of the sponge (lower case) or microbial fraction (upper case) values across species, as determined by an ANOVA on each fraction followed by an LSD multiple pairwise comparison. An asterisk indicates significantly different $\mathrm{APE}^{13} \mathrm{C}$ values between the fractions of one species. $N=$ for 5 for the microbial and sponge fractions of Acau, Aful, Cnuc, Nsub and Xboc and the microbial fraction of Nere. $N=3$ for the sponge fraction of Nere. but were all significantly less enriched compared to N. subtriangularis (Figure 1). Furthermore, unlike $N$. erecta, $\mathrm{APE}^{13} \mathrm{C}$ values of HMA species were significantly higher in light than in dark bottles, suggesting that carbon assimilation and transfer is light-dependent in these species and may be driven by photosymbiont metabolism. For all species, we observed minimal enrichment in ${ }^{13} \mathrm{C}$ in dark bottles, suggesting that heterotrophic bacteria contribute little to carbon assimilation.

Counter to our original hypothesis, however, although we observed substantial variation in nitrate assimilation and transfer across these six species (ANOVA: $P<0.001, \mathrm{~F}=56.8$ and $P<0.001$, $\mathrm{F}=17.5$ for microbial and sponge fractions, respectively), and moderate ${ }^{15} \mathrm{~N}$ enrichment of HMA species implies that these sponges host microbes capable of nitrate assimilation, N. subtriangularis was the only HMA sponge that was significantly more enriched in ${ }^{15} \mathrm{~N}$ than $N$. erecta (Figure 2). ${ }^{15} \mathrm{~N}$ enrichment was significantly lower in the dark bottles of most species (GLM: $P<0.05$; except the bacterial fraction of $A$. fulva), suggesting that nitrate assimilation may be light dependent, but that some heterotrophic assimilation in the dark may be occurring. Although our data indicate that diverse microbes, including both photosymbionts and possibly heterotrophic microbes within some sponge hosts are capable of assimilating nitrate as a source of inorganic nitrogen, regardless of irradiance, our understanding of nitrogen assimilation within marine sponges remains relatively limited (Thacker and Freeman, 2012).

Because specific symbiont groups, like cyanobacteria, are dominant members of the microbial

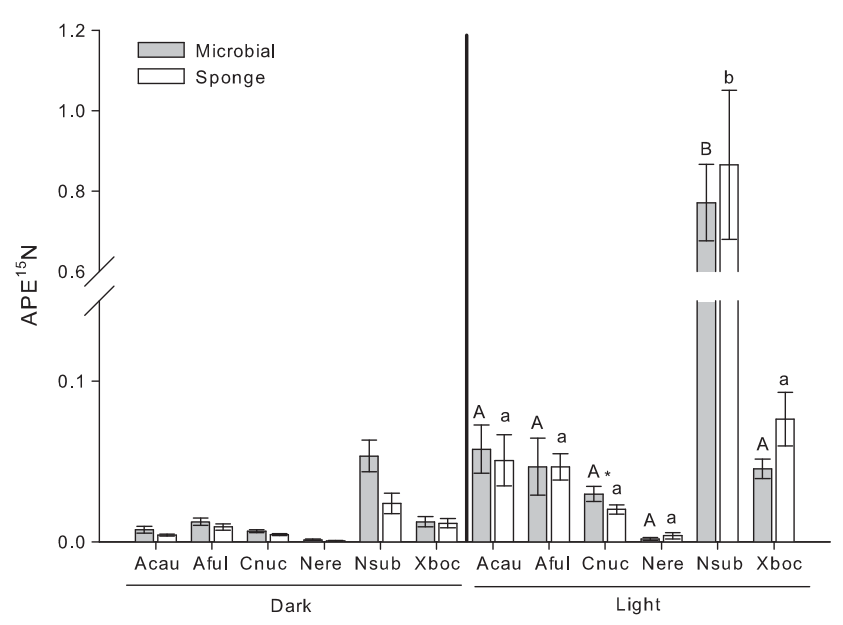

Figure 2 Mean ( \pm s.e.) $\mathrm{APE}^{15} \mathrm{~N}$ values for microbial and sponge cell fractions from six species of sponges held in light (clear) and dark (amber) bottles. Values from the dark treatment are included for reference. Different letters within columns indicate statistically significant differences in the $\mathrm{APE}^{15} \mathrm{~N}$ of the sponge (lower case) or microbial fraction (upper case) as determined by ANOVA on each fraction followed by an LSD multiple pairwise comparison. An asterisk indicates significantly different $A P E^{13} \mathrm{C}$ values between the fractions of one species. Abbreviations of species names and sample sizes are identical to Figure 1. 
consortium within many sponge species and are hypothesized to confer carbon and possibly nitrogen to the host (Taylor et al., 2007a; Usher, 2008; Freeman and Thacker, 2011), we hypothesized that variability in nutrient assimilation and transfer among host species might be driven by the abundance of photosymbionts within these hosts. As expected, photosymbiont abundance, as measured by chl $a$, varied widely across species (GLM: $P<0.001, \mathrm{~F}=237.3$; Figure 3). While N. erecta had expectedly low chl $a$ values, some HMA species (N. subtriangularis and $X$. bocatorensis) had concentrations that were well over threefold higher than other HMA species (Aplysina spp. and C. caribensis). Despite this variation across species, chl $a$ values were not strongly correlated to ${ }^{13} \mathrm{C}$ or ${ }^{15} \mathrm{~N}$ enrichment of either fraction (linear regression: $P=0.30, \mathrm{~F}=1.44, r^{2}=0.26$ and $P=0.08, \mathrm{~F}=5.65$, $r^{2}=0.59$ for $\mathrm{APE}^{13} \mathrm{C}$ of microbial and sponge fraction, respectively, and linear regression: $P=0.26, \mathrm{~F}=1.70$, $r^{2}=0.30$ and $P=0.23, \mathrm{~F}=1.99, r^{2}=0.33$ for $\mathrm{APE}^{15} \mathrm{~N}$ of microbial and sponge fraction, respectively), suggesting that photosymbiont abundance alone does not drive the observed variability of nutrient assimilation and transfer among these species. This discrepancy could be due to the presence of heterotrophic microbes that scavenge the products of photosymbiont metabolism in the light. Such heterotrophs could assimilate or transfer carbon or nitrogen, altering the ${ }^{13} \mathrm{C}$ or ${ }^{15} \mathrm{~N}$ enrichment of one or both fractions without impacting chl $a$ values. Future research is needed to determine how heterotrophic microbial symbionts affect sponge hosts.

Another common metric used to assess the status of the symbiosis between photosymbionts and host sponges is the P:R ratio. Symbiont communities that produce more energy than is required to compensate for holobiont respiration are hypothesized to provide their host sponge with a majority of its carbon

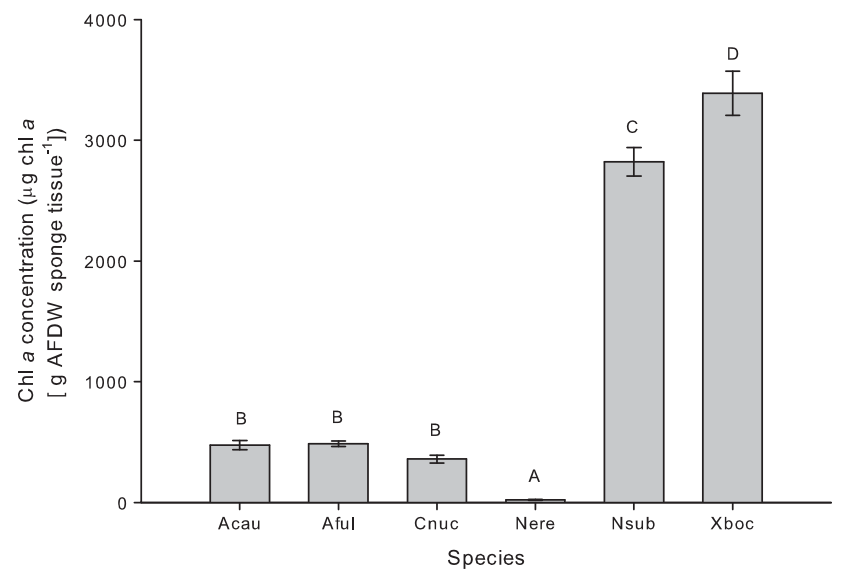

Figure 3 Mean ( \pm s.e.) chl $a$ concentrations across six species of sponges. Values are for bulk sponge tissue. Different letters above each species indicate statistically significant differences across species as determined by GLM followed by LSD multiple pairwise comparisons. Abbreviations of species names are identical to Figure 1. $N=5$ for all species. and have P:R ratios of 1.5 or above (Wilkinson, 1983; Erwin and Thacker, 2008a). By coupling our lightdark bottle incubations with DO measurements, we were able to compare the P:R ratio across species. In our incubations, only $N$. subtriangularis yielded a $P: R$ ratio large enough to be considered a phototrophic sponge. In general, P:R varied significantly across species (analysis of covariance: $P<0.001$, $\mathrm{F}=25.84$ ), with values ranging from 0.4 to 1.8 for the five species of sponges hosting dense symbiont communities, and a mean value near 0 for $N$. erecta (Figure 4). Thus, while N. subtriangularis hosts symbionts that are capable of compensating for holobiont respiration, the symbionts within other HMA species are unable to compensate for the cost of forming these symbioses. This is supported by a strong correlation between $\mathrm{P}: \mathrm{R}$ ratios and $\mathrm{APE}^{13} \mathrm{C}$ values across species (linear regression: $P=0.04$, $\mathrm{F}=9.01, r^{2}=0.69$ and $P=0.01, \mathrm{~F}=19.64, r^{2}=0.83$ for microbial and sponge fractions, respectively; Figure 5a), suggesting that not only are productive symbionts able to compensate for holobiont respiratory demands but these symbionts are also more efficient at assimilating and transferring carbon to the host. On the other hand, $\mathrm{APE}^{15} \mathrm{~N}$ values of most HMA species were low, regardless of their productivity, so $\mathrm{APE}^{15} \mathrm{~N}$ was not strongly correlated with $P: R$ ratios (linear regression: $P=0.06, F=7.12$, $r^{2}=0.64$ and $P=0.05, \mathrm{~F}=7.63, r^{2}=0.66$ for microbial and sponge fractions, respectively; Figure 5b). These data further support the hypothesis that nitrogen metabolism within these sponges may be reliant on a combination of light-mediated metabolic processes of photosymbionts and heterotrophic assimilation of nitrogen.

These trends of carbon metabolism are especially interesting given the high genetic diversity of the

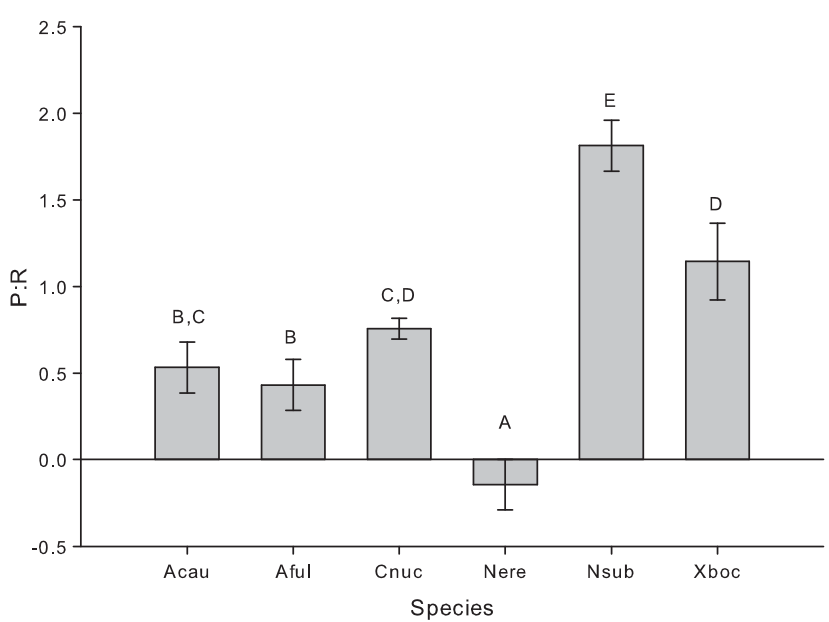

Figure 4 Adjusted least-square means ( \pm s.e.) of photosynthesis to respiration $(\mathrm{P}: \mathrm{R})$ ratios for six species of sponges. Different letters above each species indicate statistically significant differences in P:R ratios across species as determined by analysis of covariance followed by LSD multiple pairwise comparisons. Abbreviations of species names are identical to Figure 1. $N=5$ for Acau, Aful, Nere, Nsub and Xboc and $N=4$ for Cnuc. 

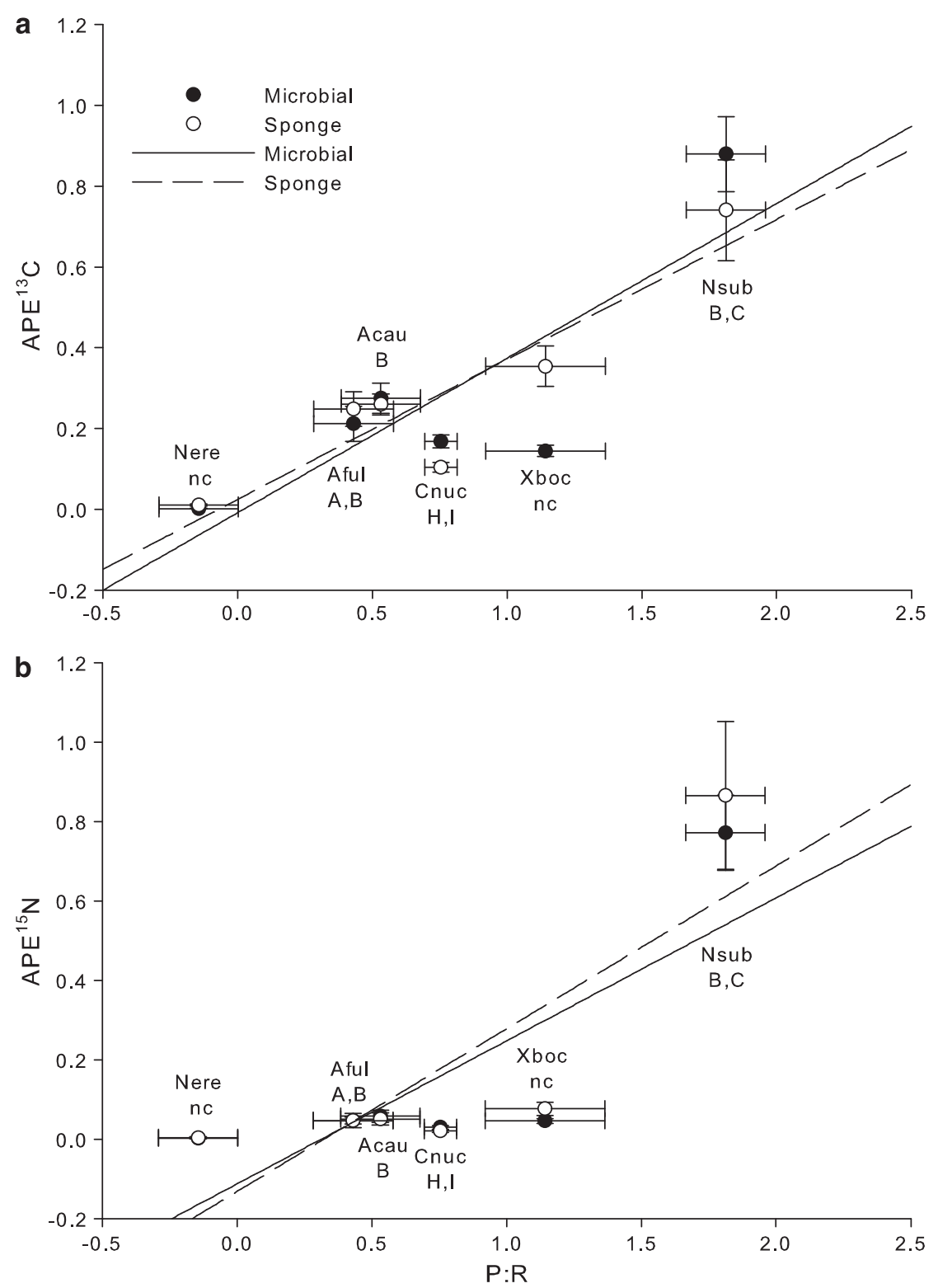

Figure 5 Sponge and microbial fraction enrichment measured in $\mathrm{APE}^{13} \mathrm{C}$ (a) and $\mathrm{APE}^{15} \mathrm{~N}$ (b) as a function of photosynthesis to respiration (P:R) ratios. $A P E^{13} \mathrm{C}$ and $A \mathrm{AE}^{15} \mathrm{~N}$ values for each fraction are means ( \pm s.e.), while P:R ratios are adjusted least-square means ( \pm s.e.). S. spongiarum clades are displayed for all sponges hosting unicellular cyanobacterial symbionts based on Erwin and Thacker (2007); nc denotes sponges that do not host $S$. spongiarum clades. Abbreviations of species names are identical to Figure 1. Sample sizes for APE and P:R ratios match those from Figures 1, 2 and 4. In each plot, the dashed line represents data from the sponge fraction and the solid line represents data from the microbial fraction.

sponge-specific cyanobacterial symbiont Synechococcus spongiarum among these hosts. Each of the species A. fulva, A. cauliformis, C. caribensis and N. subtriangularis hosts at least one unique clade of S. spongiarum (Erwin and Thacker, 2008b; Figures $5 \mathrm{a}$ and b). N. subtriangularis, A. cauliformis and A. fulva from Bocas del Toro all host $S$. spongiarum clade B, whereas N. subtriangularis hosts clade C in addition to clade B (Erwin and Thacker, 2008b Figures 5a and b). Whether increased rates of nutrient assimilation and transfer and higher productivity observed in $N$. subtriangularis is due to this unique photosymbiont clade remains unknown. If, as in corals, genetically distinct photosymbionts differ in their ability to supply the host with nutrients (LaJeunesse, 2005; Erwin and Thacker, 2008a), then the strong relationship observed between enrichment in ${ }^{13} \mathrm{C}$ and ${ }^{15} \mathrm{~N}$ and $\mathrm{P}: \mathrm{R}$ (Figure 5) may be generated by photosymbiont diversity across species (Erwin and Thacker, 2008b; Freeman and Thacker, 2011).

If genetically distinct symbionts assimilate nutrients and transfer them at different rates, then one might expect to measure differences in the mean $A P E^{13} \mathrm{C}$ and $A P E^{15} \mathrm{~N}$ values between the microbial and host cell fractions of each species. In contrast, we measured similar values for the two fractions in most species, with the only exceptions being the 
microbial fraction of $C$. caribensis, which was significantly enriched in ${ }^{13} \mathrm{C}$ and ${ }^{15} \mathrm{~N}$ relative to the sponge fraction (GLM: $P=0.003, \quad F=38.51$ and $P=0.009, \quad \mathrm{~F}=22.9$ for ${ }^{13} \mathrm{C}$ and ${ }^{15} \mathrm{~N}$, respectively; Figure 1) and the sponge fraction of $X$. bocatorensis, which was enriched in ${ }^{13} \mathrm{C}$ relative to the microbial fraction (GLM: $P=0.007, \quad F=25.4$ ). While these observations might suggest that the symbiosis in C. caribensis is less efficient than that in X. bocatorensis or that $X$. bocatorensis might be able to stimulate nutrient transfer from its symbionts, future work is needed to explicitly test these hypotheses. Such studies should quantify productivity, nutrient assimilation and transfer to the host at a wider variety of time scales than reported here to assess the lag time between symbiont and host enrichment.

It is not surprising that the results from this study do not exactly mirror the trends for the Aplysina spp., $N$. subtriangularis and $N$. erecta seen in Freeman and Thacker (2011). For instance, using natural abundance isotope values, we have shown that, in nature, $A$. cauliformis obtains both $\mathrm{C}$ and $\mathrm{N}$ from its symbionts, while $A$. fulva obtains only $\mathrm{N}$ and $N$. subtriangularis obtains only $\mathrm{C}$ from its symbiont community and $N$. erecta obtains both $\mathrm{C}$ and $\mathrm{N}$ from particulate organic matter (Freeman and Thacker, 2011). These $\delta^{13} \mathrm{C}$ and $\delta^{15} \mathrm{~N}$ ratios represent natural, integrated records detailing whether these sponges are assimilating their $\mathrm{C}$ and/or $\mathrm{N}$ from particulate organic matter or from symbiont sources over time. These values thus account for the fact that nutrient exchange may be due to factors such as ambient nutrient levels, particulate organic matter feeding, nutrient recycling within the symbiosis or other currently unknown factors. These light-dark bottle incubations, on the other hand, are assessments of how efficient the symbionts within these sponges are at assimilating and transferring nutrients when supplied with low, but non-limiting, levels of Cand $\mathrm{N}$-based compounds enriched in ${ }^{13} \mathrm{C}$ and ${ }^{15} \mathrm{~N}$. Comparisons of data from across these two studies, however, may help to explain some of these trends.

Nutrient assimilation and transfer to the host may be driven more by symbiont productivity and phylotype than by symbiont abundance, implying that that these relationships are complex and tightly coevolved symbioses (Knowlton and Rohwer, 2003; Thacker and Freeman, 2012). However, it remains unclear why some sponges host less productive clades and what processes might prevent a wider distribution of the potentially more productive $S$. spongiarum clade C. In a tightly coevolved relationship, selection might favor sponge-microbe symbioses with minimal cost:benefit ratios from the host's perspective. Because both heterotrophic filter feeding and abundant communities of autotrophic symbionts present metabolic costs to the host sponge, we hypothesize that the host will balance these two feeding modes to receive sufficient nutrients at minimal metabolic cost. For instance, $N$. subtriangularis hosts the highly productive combination of clades B and C, which provides the host with more carbon than is required to compensate for holobiont respiration. The cost of hosting these symbionts might be reflected in lower heterotrophic feeding, as this sponge assimilates $<25 \%$ of its carbon from filter-feeding (Freeman and Thacker 2011). However, A. fulva hosts the less productive clades $\mathrm{A}$ and $\mathrm{B}$, and cannot compensate for holobiont respiration using symbiont-derived nutrition alone; reduced autotrophy might drive increased heterotrophy, as this host assimilates over $50 \%$ of its carbon from filter-feeding (Freeman and Thacker, 2011). Indeed, these trade-offs between autotrophic and heterotrophic feeding modes appear to be well established; even under shaded conditions, $N$. subtriangularis maintains significant assimilation of symbiont-derived carbon, while A. fulva becomes more reliant on heterotrophic feeding (Freeman and Thacker, 2011).

Although abundant populations of microbial symbionts are present within many sponge species, the relative benefits of these symbioses are driven more by the presence or absence of particular groups of symbionts than overall symbiont abundance. These findings imply that the host may exert a level of control over symbiont community composition in an attempt to maximize the benefits and minimize the costs associated with different symbiont phylotypes (Bronstein, 2001; Cowden and Peterson, 2009; Thacker and Freeman, 2012).

${ }^{13} \mathrm{C}$ assimilation and transfer under varying irradiance Our coupling of short-term incubations with enriched isotope tracers can be readily adapted to examine how nutrient exchange differs under changing environmental conditions. Given that A. cauliformis maintains high levels of symbiontderived carbon inputs, even under shaded conditions (Freeman and Thacker, 2011), we chose this species to test the hypothesis that symbiont carbon fixation and nutrient transfer to the host sponge would vary across discrete irradiance levels and would be directly related to productivity. As expected, two and five layers of shade cloth provided enough light to be near (0.8) and well below (0.17) the compensation point (Erwin and Thacker, 2008a). Taken together with the light bottles, these treatments provided three distinct light environments, and we observed three distinct P:R ratios (GLM for treatment: $P<0.001, F=49.8$; Figure 6a). As hypothesized, carbon fixation and transfer differed across light treatments (GLM for treatment: $P=0.001, \mathrm{~F}=11.52$ and $P=0.001, F=11.6$ for microbial and sponge fractions, respectively), was highest at the maximum irradiance (Figure 6b), and was correlated with P:R ratio (GLM testing the effects of individual and P:R ratio on $\mathrm{APE}^{13} \mathrm{C}: P<0.01, \mathrm{~F}=16.6, r^{2}=0.62$ and $P<0.001, \mathrm{~F}=21.7, r^{2}=0.69$ for microbial and sponge fractions, respectively). In fact, while the sponge fraction was approximately $50 \%$ as enriched as the 
1124
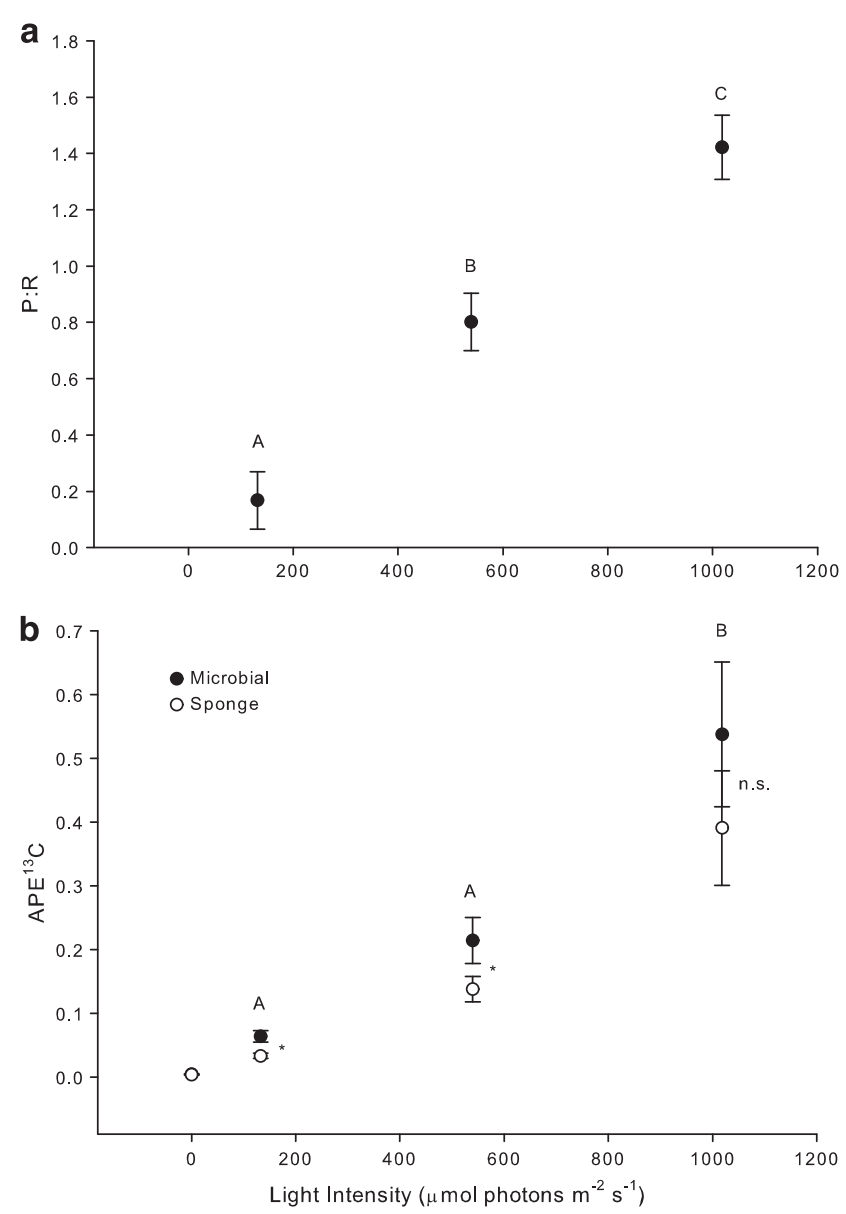

Figure 6 Mean ( \pm s.e.) photosynthesis to respiration (P:R) ratios (a) and mean ( \pm s.e.) $\mathrm{APE}^{13} \mathrm{C}$ values for microbial and sponge cell fractions (b) of $A$. cauliformis incubated under three different irradiances. Different letters indicate statistically significant differences across treatments as determined by GLM followed by an LSD multiple pairwise comparison. In (b), differences across treatments were identical for sponge and microbial fractions, so only one letter is shown to represent both fractions of each treatment. Dark values were not included in the analysis but are shown for reference. An asterisk indicates statistically significant differences in mean $\mathrm{APE}^{13} \mathrm{C}$ between sponge and microbial fractions, while NS signifies no significant difference between fractions within treatment, as determined by GLM on each treatment. $N=7$ for full light and lightly shaded treatment and $N=8$ for heavily shaded treatment (a) and $N=8$ for the sponge and microbial fractions of dark, heavily shaded and full light treatments and $N=7$ for both fractions in the lightly shaded treatment (b).

bacterial fraction under heavy shade, this increased to 66 and $80 \%$ under light shade and maximum irradiance treatments, respectively, suggesting that the efficiency of nutrient exchange increased with irradiance and that, at low light, photosymbionts were limiting their transfer of carbon to the sponge host (ANOVA comparing heavy shade and maximum irradiance treatments: $P=0.03, \mathrm{~F}=2.14$ ). This is also evident when comparing the $\mathrm{APE}^{13} \mathrm{C}$ values of the microbial and sponge cell fractions; the microbial cell fraction was more enriched than the sponge fraction in the shaded treatments (GLM on fraction: $P=0.01, \mathrm{~F}=12.86$ and $P<0.001, \mathrm{~F}=32.06$ for lightly and heavily shaded, respectively), but these fractions were similar in the maximum irradiance treatment (GLM on fraction: $P=0.13, F=2.98$; Figure 6b). These data represent the first assessment of symbiont carbon fixation and transfer in a sponge species under differing environmental conditions. These results demonstrate that, within an individual host species, productivity not only varies with irradiance but also is directly related to carbon assimilation and transfer. Future studies are needed to determine how these processes vary among host species. For instance, because the closely related species $A$. fulva appears to host symbionts, but obtains minimal carbon nutrition from them (Freeman and Thacker, 2011), similar incubations at different irradiances might yield a gradient of enrichment in the microbial fraction, whereas the enrichment of the sponge fraction remains relatively static.

Our results suggest that genetically distinct photosymbiont clades differ in their productivity and their ability to assimilate and transfer carbon to the host sponge. These findings have important implications for the understanding of metazoanmicrobe symbioses. It is frequently assumed that abundant microbial communities benefit their host but, in reality, our ability to delineate between mutualistic, commensal or parasitic microbes is minimal (Thacker and Freeman, 2012). Our study highlights the fact that abundant microbial communities are not equally beneficial to all hosts. Such disparity across hosts appears to be due more to the quality than the quantity of the symbionts, with a particular emphasis on the presence or absence of particular symbiont groups and holobiont productivity. While it is apparent that studies coupling molecular methods with manipulative experiments in sponge-microbe symbioses lag behind those of other model systems (Ruby, 2008), future studies using cultured photosymbionts have the potential to greatly increase our understanding of these interactions. For instance, infection and colonization experiments in which host sponges at various life stages are exposed to genetically diverse symbionts may allow us to determine what factors (that is, selective provisioning of nutrients to certain phylotypes by the host sponge or the pairing of symbiont phylotypes with host sponges based on symbiont nutrient dependency) impact these symbioses.

\section{Acknowledgements}

We thank R Collin, G Jacome and P Gondola at the Smithsonian Tropical Research Institute Bocas Research Station and Roxane Bowden, Geophysical Laboratory for technical assistance. Financial support for this project came from a Smithsonian Tropical Research Institute Short-Term Fellowship, a Sigma Xi Grants-in-Aid of Research to CJF, US National Science Foundation Grants 0726944 and 0829986 to RWT and a Smithsonian Institution Marine Science Network Postdoctoral Fellowship awarded to DMB; the Carnegie Institution of Washington to DMB and MLF, and the WM Keck Foundation (072000) to MLF. 


\section{References}

Becerro MA, Paul VJ. (2004). Effects of depth and light on secondary metabolites and cyanobacterial symbionts of the sponge Dysidea granulosa. Mar Ecol Prog Ser 280: $115-128$.

Becerro MA. (2008). Quantitative trends in sponge ecology research. Mar Ecol 29: 67-177.

Boucher DH, James S, Keeler KH. (1982). The ecology of mutualism. Annu Rev Ecol Syst 13: 315-347.

Bronstein JL. (2001). The costs of mutualism. Amer Zool 41: 825-839.

Cowden CC, Peterson CJ. (2009). A multi-mutualist simulation: applying biological market models to diverse mycorrhizal communities. Ecol Model 220: 1522-1533.

Erwin PM, Thacker RW. (2007). Incidence and identity of photosynthetic symbionts in Caribbean coral reef sponge assemblages. J Mar Biol Ass UK 87: 1683-1692.

Erwin PM, Thacker RW. (2008a). Phototrophic nutrition and symbiont diversity of two Caribbean sponge-cyanobacteria symbioses. Mar Ecol Prog Ser 362: 139-147.

Erwin PM, Thacker RW. (2008b). Cryptic diversity of the symbiotic cyanobacterium Synechococcus spongiarum among sponge hosts. Mol Ecol 17: 2937-2947.

Freeman CJ, Thacker RW. (2011). Complex interactions between marine sponges and their symbiotic microbial communities. Limnol Oceanogr 56: 1577-1586.

Fry B. (2006). Stable Isotope Ecology. Springer: New York.

Hentschel U, Usher KM, Taylor MW. (2006). Marine sponges as microbial fermenters. FEMS Microbiol Ecol 55: $167-177$.

Hill R, Scott A. (2012). The influence of irradiance on the severity of thermal bleaching in sea anemones that host anemonefish. Coral Reef 31: 273-284.

Knowlton N, Rohwer F. (2003). Multispecies microbial mutualisms on coral reefs: the host as a habitat. $\mathrm{Am}$ Nat 162: S51-562.

LaJeunesse TC. (2005). "Species" radiations of symbiotic dinoflagellates in the Atlantic and Indo-Pacific since the Miocene-Pliocene transition. Mol Biol Evol 22: 570-581.

Moya A, Pereto J, Gil R, Latorre A. (2008). Learning how to live together: genomic insights into prokaryote-animal symbioses. Nat Rev 9: 218-228.

Muscatine L, Cernichiari E. (1969). Assimilation of photosynthetic products of zooxanthellae by a reef coral. Biol Bull 137: 506-523.

Muscatine L, Porter JW, Kaplan IR. (1989). Resource partitioning by reef corals as determined from stable isotope composition I. $\delta^{13} \mathrm{C}$ of zooxanthellae and animal tissue vs depth. Mar Biol 100: 185-193.

Nyholm SV, McFall-Ngai MJ. (2004). The winnowing: establishing the squid-Vibrio symbiosis. Nat Rev Microbiol 2: 623-642.

Rosenberg E, Sharon G, Atad I, Zilber-Rosenberg I. (2010). The evolution of animals and plants via symbiosis with microorganisms. Environ Microbiol Rep 2: 500-506.

Ruby EG. (2008). Symbiotic conversations are revealed under genetic interrogation. Nat Rev Microbiol 6: 752-762.

Sacristan-Soriano O, Banaigs B, Casamayor EO, Becerro MA. (2011). Exploring the links between natural products and bacterial assemblages in the sponge Aplysina aerophoba. Appl Environ Microbiol 77: 862-870.

Siegl A, Kamke J, Hochmuth T, Piel J, Richter M, Liang C et al. (2011). Single-cell genomics reveals the lifestyle of Poribacteria, a candidate phylum symbiotically associated with marine sponges. ISME J 5: 61-70.

Tanaka Y, Miyajima T, Koike I, Hayashibara Y, Ogawa H. (2006). Translocation and conservation of organic nitrogen within the coral-zooxanthella symbiotic system of Acropora pulchra, as demonstrated by dual isotope-labeling techniques. J Exp Mar Biol Ecol 336: 110-119.

Taylor MW, Hill RT, Piel J, Thacker RW, Hentschel U. (2007b). Soaking it up: the complex lives of marine sponges and their microbial associates. ISME J 1: 187-190.

Taylor MW, Radax R, Steger D, Wagner M. (2007a). Sponge-associated microorganisms: evolution, ecology, and biotechnological potential. Microbiol Mol Biol Rev 71: 295-347.

Thacker RW, Diaz MC, Rutzler K, Erwin PM, Kimble SJA, Pierce MJ et al. (2007). Phylogenetic relationships among the filamentous cyanobacterial symbionts of Caribbean sponges and a comparison of photosynthetic production between sponges hosting filamentous and unicellular cyanobacteria. In: Hajdu E, Muricy G (eds) Porifera Research: Biodiversity, Innovation, and Sustainability. Museu Nacional: Rio de Janeiro, pp 621-626.

Thacker RW, Freeman CJ. (2012). Sponge-microbe symbioses: recent advances and new directions. In: Becerro MA, Uriz MJ, Maldonado M, Turon X (eds), Advances in Sponge Science: Phylogeny, Systematics, Ecology. Advances in Marine Biology Vol. 62. Elsevier: Amsterdam, pp 57-111.

Usher KM. (2008). The ecology and phylogeny of cyanobacterial symbionts in sponges. Mar Ecol 29: 178-192.

Venn AA, Loram JE, Douglas AE. (2008). Photosynthetic symbioses in animals. J Exp Bot 59: 1069-1080.

Vrijenhoek RC. (2010). Genetics and evolution of deep-sea chemosynthetic bacteria and their invertebrate hosts. In: Kiel S (ed), The Vent and Seep Biota, Topics in Geobiology. Springer: Berlin, pp 15-49.

Webster NS, Blackall LL. (2009). What do we really know about sponge-microbial symbioses? ISME J 3: 1-3.

Webster NS, Taylor MW, Behnam F, Lücker S, Rattei T, Whalan S et al. (2010). Deep sequencing reveals exceptional diversity and modes of transmission for bacterial sponge symbionts. Environ Microbiol 12: 2070-2082.

Webster NS, Taylor MW. (2012). Marine sponges and their microbial symbionts: love and other relationships. Environ Microbiol 14: 335-346.

Weisz JB, Hentschel U, Lindquist N, Martens CS. (2007). Linking abundance and diversity of sponge-associated microbial communities to metabolic differences in host sponges. Mar Biol 152: 475-483.

Weisz JB, Massaro AJ, Ramsby BD, Hill MS. (2010). Zooxanthellar symbionts shape host sponge trophic status through translocation of carbon. Biol Bull 219: 189-197.

Wilkinson CR. (1983). Net primary productivity in coral reef sponges. Science 219: 410-412.

Zilber-Rosenberg I, Rosenberg E. (2008). Role of microorganisms in the evolution of animals and plants: the hologenome theory of evolution. FEMS Microbiol Rev 32: $723-735$.

Supplementary Information accompanies the paper on The ISME Journal website (http://www.nature.com/ismej) 